\title{
Age, period and cohort analysis of patient dental visits in Australia
}

\author{
Xiangqun Ju, David S Brennan* and A John Spencer
}

\begin{abstract}
Background: Understanding dentists' capacity to supply dental services over time is a key element in the process of planning for the future. The aim was to identify time trends and estimate age, period and cohort effects in patients' visits supplied per dentist per year.

Methods: Mailed questionnaires were collected from a random sample of Australian private general practice dentists. The response rates were 73\%, 75\%, 74\%, 71\%, 76\% and 67\% in 1983, 1988, 1993, 1998, 2003 and 2009, respectively. The time trends in the mean number of patient visits supplied per dentist per year (PPY) was described by using a standard cohort table and age-period-cohort analyses applying a nested general linear regression models approach.
\end{abstract}

Results: The mean number of PPY decreased across most age groups of dentists over the time of study. The age-period model showed that younger dentists (20-29 years) and older dentists (65-74 and 80-84 years) had lower PPY than middle-aged dentists, and the age-cohort model showed higher PPY among earlier cohorts, and lower PPY among more recent cohorts.

Conclusion: The study found a period effect of declining PPY over the observation period. More recent cohorts of dentists provide lower numbers of PPY than earlier cohorts at similar ages, but the provision of PPY among these younger cohorts appeared to be stable as they moved into middle age.

Keywords: Age, Period, Cohort, Patient visits

\section{Background}

In Australia the majority of practising dentists work in the private sector $(83 \%)$, with dental services being provided mainly by general dental practitioners (85\%) [1]. Dental services are generally provided on a fee-for-service basis, paid either directly by the individual or indirectly through private insurance.

The capacity of practising dentists to supply dental services has not only been linked with population demographics and oral health status, but also associated with labour force structure and service-mix provided. To measure practice activity provided by dentists, the time measure of the hours per dentist per year (HPY) and the patient measure of the number of patient visits per dentist per hour $(\mathrm{PPH})$ were used to produce the measure of patient visits per dentist per year $(\mathrm{PPY}=\mathrm{HPY} \times \mathrm{PPH})$

\footnotetext{
* Correspondence: david.brennan@adelaide.edu.au

Australian Research Centre for Population Oral Health, The University of Adelaide, South Australia 5005, Australia
}

as in previous research [1]. PPY was adopted as a key marker of dentist's capacity to provide services and has shown a decreasing trend over time in Australia [2-4].

Age, period and cohort effects are important considerations when explaining trends in patient visits supplied by practising dentists [5]. Age effects are associated with the passage of time, so change in the number of patient visits by practising dentists related to age over time may help to explain the capacity to provide dental service, for instance, if dentists became less productive as they aged. Period effects can affect all ages simultaneously over time. For example, they can mark the occurrence of a particular historical event, such as the availability of modern highspeed electric dental handpieces. Cohort effects involve changes across groups with the same birth year who experience the same event during the same period. There is a linear dependency between age, period and cohort, because age, period and cohort membership is predicted by any two of the three effects. Therefore, it is difficult to 
estimate the three separate effects. A nested models approach can be used to estimate and assess the fit of different models [6,7], such as age-period and agecohort models.

Understanding trends over time in the supply of patient visits and the relationship with labour force age structure and possible cohort effects is important in dental labour force planning, informing current policies and projections of future capacity to supply services. The aim of this study was to identify trends in patients visits supplied by practising dentists in Australia, and estimate age, period and cohort effects in PPY over an observation period spanning 1983 to 2010 .

\section{Method}

\section{Data collection}

The data were from the Longitudinal Study of Dentists' Practice Activity, which is designed to provide estimates of dentist practices and service provision of Australian private general practising dentists over time. The study was approved by the ethics committee of the Australian Institute of Health and Welfare (AIHW).

Details of the methods have been previously described [1,8-10]. Briefly, a random sample of $10 \%$ of male and $40 \%$ of female dentists was selected from the dental registers for each State or Territory in Australia in 1983-84, 1988-89, 1993-94, 1998-99, 2003-04 and 2009-10 as waves of a longitudinal study. All sampled dentists from previous waves of the study were included again at each successive wave. Sample supplementation of newly registered male and female dentists at each successive wave was also used to add to the sample to ensure representative cross-sectional estimates.

These dentists were surveyed by mailed questionnaire. The practising dentists provided estimates of the number of patients treated per day, and the number of hours per day, days per week and weeks per year spent working. From this information, practice activity measures were calculated as follows:

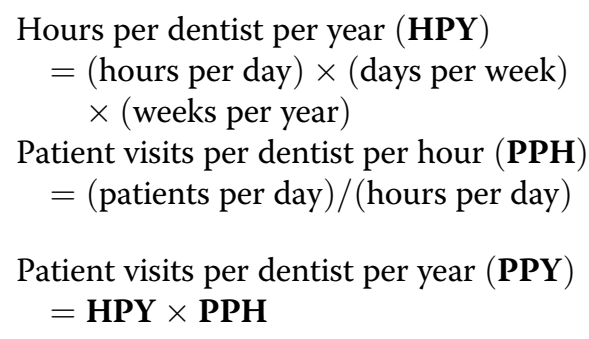

\section{Weighting}

The data were weighted prior to analysis. The data were weighted using numbers of private general practising dentists at December 1983 and 1988, with age and sex distributions of dentists from the 1981 and 1986 population censuses of Australia, and dental board registration statistics from 1992, 1994, 2000 and 2009 [1,3,8-10]. The weights adjusted the sample to the age-specific population distribution of male and female dentists in the dentist population.

\section{Analysis}

All sampled dentists were included and the analysis treated the sample as a synthetic cohort as this maintained representative cross-sectional estimates at each point in time rather than restricting analyses only to longitudinal cases. A standard cohort table was produced to provide an initial description of the effects of age, period and cohort by creating 5-year age groups and corresponding 5-year birth cohorts. Age group formed the rows of the table and time period formed the columns, which provided synthetic 5-year birth cohorts in each diagonal running downwards from left to right across the table [5].

General linear regression was applied to estimate mean number of PPY by age, period and cohort factors using SAS statistical software (SAS 9.2). A set of nested models were examined for goodness-of-fit, and F-tests were applied to determine which models were preferred [11]. The details are described below.

The age model was used as a starting point in analysis and followed by age-drift, age-period, age-cohort and age-period-cohort models [12]. The age model was made up of 14 age groups coded as indicator (dummy) variables. The age-drift model consisted of the 14 variables for age (coded from 1 to 14), plus the six time periods (coded from 1 to 6) entered as a continuous variable to model regular trends not ascribed to either period or cohort influence. This variation is referred to as "drift" $[6,7,12]$. The age-period model consisted of the 14 indicator variables for age, with period entered as indicator variables. The age-cohort model consisted of the 14 indicator variables for age, combined with 17 indicator variables for dentist birth cohort. The age-period-cohort model consisted of the indicator variables for age, period and cohort.

Goodness-of-fit tests were applied for each model and F-tests were used to assess the models. The models with a good fit to data were pursued further to provide a parsimonious explanation of the data.

\section{Results}

The response rates were $73 \%, 75 \%, 74 \%, 71 \%, 76 \%$ and 67\% in 1883-84, 1988-89, 1993-94, 1998-99, 2003-4 and $2009-10$, respectively.

Table 1 is a standard cohort table which presents age distributions of private general practice dentists by time of data collection. Five-year age groups were coded into 
Table 1 Number of dentists by age group and time of data collection (unweighted data)

\begin{tabular}{|c|c|c|c|c|c|c|c|c|c|c|c|c|c|c|}
\hline \multirow{2}{*}{$\begin{array}{l}\text { Age group } \\
\text { (years) }\end{array}$} & \multicolumn{2}{|c|}{1983} & \multicolumn{2}{|c|}{1988} & \multicolumn{2}{|c|}{1993} & \multicolumn{2}{|c|}{1998} & \multicolumn{2}{|c|}{2003} & \multicolumn{2}{|c|}{2009} & \multicolumn{2}{|c|}{ All } \\
\hline & $N$ & $(\%)$ & $\mathbf{N}$ & $(\%)$ & $\mathbf{N}$ & $(\%)$ & $\mathrm{N}$ & $(\%)$ & $N$ & $(\%)$ & $\mathrm{N}$ & $(\%)$ & $\mathbf{N}$ & (\%) \\
\hline $20-24$ & 6 & 1.7 & 24 & 5.2 & 18 & 4.2 & 15 & 3.3 & 17 & 3.3 & 16 & 2.6 & 96 & 3.4 \\
\hline $25-29$ & 62 & 17.8 & 77 & 16.7 & 62 & 14.6 & 69 & 15.4 & 60 & 11.8 & 98 & 15.6 & 428 & 15.2 \\
\hline $30-34$ & 64 & 18.3 & 94 & 20.4 & 69 & 16.2 & 62 & 13.8 & 78 & 15.3 & 107 & 17.1 & 474 & 16.8 \\
\hline $35-39$ & 49 & 14.0 & 73 & 15.8 & 86 & 20.2 & 84 & 18.7 & 62 & 12.2 & 87 & 13.9 & 441 & 15.6 \\
\hline $40-44$ & 43 & 12.3 & 49 & 10.6 & 55 & 12.9 & 80 & 17.8 & 98 & 19.2 & 64 & 10.2 & 389 & 13.8 \\
\hline $45-49$ & 29 & 8.3 & 46 & 10.0 & 48 & 11.3 & 54 & 12.0 & 84 & 16.5 & 78 & 12.4 & 339 & 12.0 \\
\hline $50-54$ & 27 & 7.7 & 27 & 5.9 & 31 & 7.3 & 37 & 8.2 & 54 & 10.6 & 82 & 13.1 & 258 & 9.1 \\
\hline $55-59$ & 40 & 11.5 & 26 & 5.6 & 19 & 4.5 & 24 & 5.4 & 29 & 5.7 & 45 & 7.2 & 183 & 6.5 \\
\hline $60-64$ & 16 & 4.6 & 27 & 5.9 & 18 & 4.2 & 9 & 2.0 & 14 & 2.8 & 34 & 5.4 & 118 & 4.2 \\
\hline $65-69$ & 7 & 2.0 & 11 & 2.4 & 13 & 3.1 & 4 & 0.9 & 8 & 1.6 & 12 & 1.9 & 55 & 1.9 \\
\hline $70-74$ & 4 & 1.2 & 5 & 1.1 & 3 & 0.7 & 10 & 2.2 & 2 & 0.4 & 3 & 0.5 & 27 & 1.0 \\
\hline $75-79$ & 1 & 0.3 & 0 & 0.0 & 3 & 0.7 & 0 & 0.0 & 4 & 0.8 & 0 & 0.0 & 8 & 0.3 \\
\hline $80-84$ & 1 & 0.3 & 1 & 0.2 & 0 & 0.0 & 1 & 0.2 & 0 & 0.0 & 1 & 0.2 & 4 & 0.1 \\
\hline $85-89$ & 0 & 0.0 & 1 & 0.2 & 0 & 0.0 & 0 & 0.0 & 0 & 0.0 & 0 & 0.0 & 1 & 0.0 \\
\hline Total & 349 & 100. & 461 & 100 & 425 & 100 & 449 & 100 & 510 & 100 & 627 & 100. & 2821 & 100 \\
\hline
\end{tabular}

14 categories, combined with five-yearly periods (six years interval for 2003 to 2009), which provided synthetic 5-year birth cohorts of practising dentists in each diagonal running downwards from left to right across the table.

Higher numbers of practicing dentists were observed in the 25-29 to 60-64 year age groups. Cell sizes of less than 10 occurred in the youngest (20-24 years) or 65 years and older groups at some points in time. Dental practice activity for these smaller groups should be interpreted with caution.

Mean number and standard error of PPY by age and year of study are showed in Table 2 and presented graphically in Figures 1 and 2. Figure 1 shows mean number of PPY by age and year of study. In general, mean number of PPY decreased across most age groups over the time of study. PPY was lower in younger age groups (less than 25 years) and older age groups (65 years or older groups), and tended to be higher in middle age groups (30-34 to 60-64 year age groups). Figure 2 shows mean number of PPY by dentist age, year of study and birth cohort. Each line represents a dentist 5-year birth cohort over the times of data collection. Most cohorts are represented by six observation times.

The fit of a set of age, age-drift, age-period, agecohort, and age-period-cohort models were assessed for the mean number of PPY. R-squared and P-values from these models are showed in Table 3. A P-value of 0.05 is taken as the significance level, and higher R-squared indicated a better model fit. R-squared increased with more complex models, such as the age drift model's R-squared was increased over 50\% compared to the age model. However, R-squared showed little change from age-drift, age-period or age-cohort models to the ageperiod-cohort model.

F-tests were applied to test age, period and cohort effects in the nested models, which compared the age versus age-drift, then age-drift versus age-period and age-cohort, then age-period and age-cohort versus ageperiod-cohort models. F and P values from F- tests are presented in Table 4.

While the age-period-cohort model provided the best fit to the data, the age, period and cohort effects are not completely independent. The age-period and age-cohort models were therefore examined in order to interpret the effects of age, period and cohort on PPY.

Table 5 presents the parameter estimates and standard error of mean number of PPY from general linear regression for the age-period and age-cohort models. A parameter estimate of 0 indicated which group was the reference group. A parameter estimate less than 0 indicated a lower average number of PPY and greater than 0 indicated higher average number of PPY, compared with the reference group. The reference categories used were the 30-34 year age group, the data collection from 2009-10 and the dentist birth cohort that was aged 25-29 years in 1983 .

In the age-period model, the age effect showed that compared to the reference group of 30-34 years there were negative parameter estimates indicating lower PPY in younger (20-24 and 25-29 years) and older age groups (65-69, 70-74 and 80-84 years). Age groups 40-44 and 50-54 years had positive parameter estimates indicating higher PPY. The period effect showed that compared to the reference group of 2009, the periods 
Table 2 Mean number and standard error of PPY by age group and time of data collection (weighted)

\begin{tabular}{|c|c|c|c|c|c|c|c|c|c|c|c|c|c|c|}
\hline \multirow{2}{*}{$\begin{array}{l}\text { Age group } \\
\text { (years) }\end{array}$} & \multicolumn{2}{|c|}{1983} & \multicolumn{2}{|c|}{1988} & \multicolumn{2}{|c|}{1993} & \multicolumn{2}{|c|}{1998} & \multicolumn{2}{|c|}{2003} & \multicolumn{2}{|c|}{2009} & \multicolumn{2}{|c|}{ All } \\
\hline & Mean & SE & Mean & SE & Mean & SE & Mean & SE & Mean & SE & Mean & SE & Mean & SE \\
\hline $20-24$ & 2103 & 356 & 2365 & 163 & 2789 & 236 & 1550 & 199 & 2301 & 262 & 2033 & 186 & 2231 & 96 \\
\hline $25-29$ & 3005 & 151 & 2743 & 123 & 2853 & 146 & 2375 & 111 & 2505 & 138 & 2172 & 89 & 2617 & 53 \\
\hline $30-34$ & 3902 & 200 & 3048 & 137 & 2638 & 155 & 2662 & 166 & 2472 & 128 & 2211 & 113 & 2772 & 64 \\
\hline $35-39$ & 3807 & 200 & 3565 & 177 & 2790 & 132 & 2435 & 109 & 2402 & 152 & 2440 & 128 & 2845 & 64 \\
\hline $40-44$ & 3506 & 238 & 3326 & 190 & 3496 & 162 & 2772 & 144 & 2652 & 118 & 2630 & 228 & 2979 & 73 \\
\hline $45-49$ & 3425 & 314 & 3199 & 212 & 2899 & 173 & 3033 & 157 & 2788 & 123 & 2410 & 128 & 2859 & 69 \\
\hline $50-54$ & 3267 & 208 & 3531 & 332 & 3036 & 269 & 2884 & 225 & 2746 & 154 & 2529 & 135 & 2889 & 84 \\
\hline $55-59$ & 3533 & 303 & 3218 & 177 & 2870 & 241 & 2660 & 305 & 2904 & 183 & 2682 & 233 & 3005 & 106 \\
\hline $60-64$ & 3529 & 409 & 2952 & 335 & 2354 & 171 & 2805 & 350 & 2233 & 348 & 2404 & 156 & 2603 & 115 \\
\hline $65-69$ & 2424 & 667 & 1835 & 257 & 1818 & 292 & 1499 & 343 & 1249 & 273 & 2779 & 492 & 2000 & 185 \\
\hline $70-74$ & 2030 & 466 & 2833 & 945 & 888 & 168 & 1961 & 377 & 1115 & 425 & 1821 & 1043 & 1891 & 256 \\
\hline $75-79$ & 1000 & & & & 1871 & 818 & & & 2518 & 1028 & 648 & & 2200 & 602 \\
\hline $80-84$ & & & 490 & & & & 320 & & & & & & 476 & 103 \\
\hline $85-89$ & & & 1000 & & & & & & & & & & 1000 & \\
\hline Total & 3405 & 82 & 3097 & 65 & 2816 & 61 & 2589 & 58 & 2550 & 52 & 2418 & 51 & 2762 & 25 \\
\hline
\end{tabular}

1983 to 1998 had positive parameter estimates indicating higher PPY.

In the age-cohort model, the age effect showed that the age groups $45-49$ to $80-84$ years all had negative parameter estimates indicating lower PPY than the reference group of 30-34 years. The cohort effect showed that compared to the reference group of 25-29 years in 1983, older cohorts aged 30-34 to 70-74 years in 1983 had positive parameter estimates indicating higher PPY, while younger cohorts aged 20-24 in 1983 to $20-24$ years

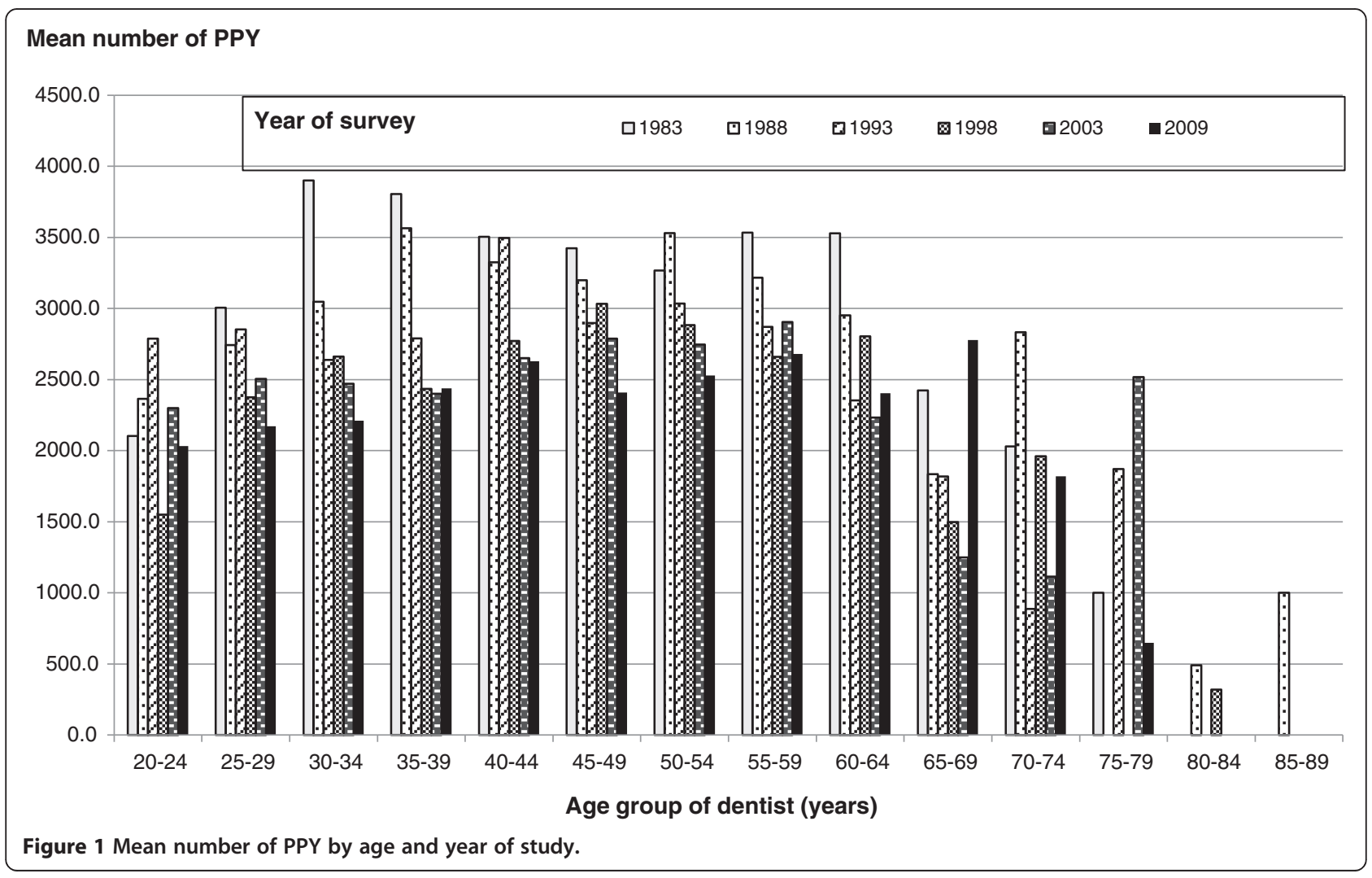




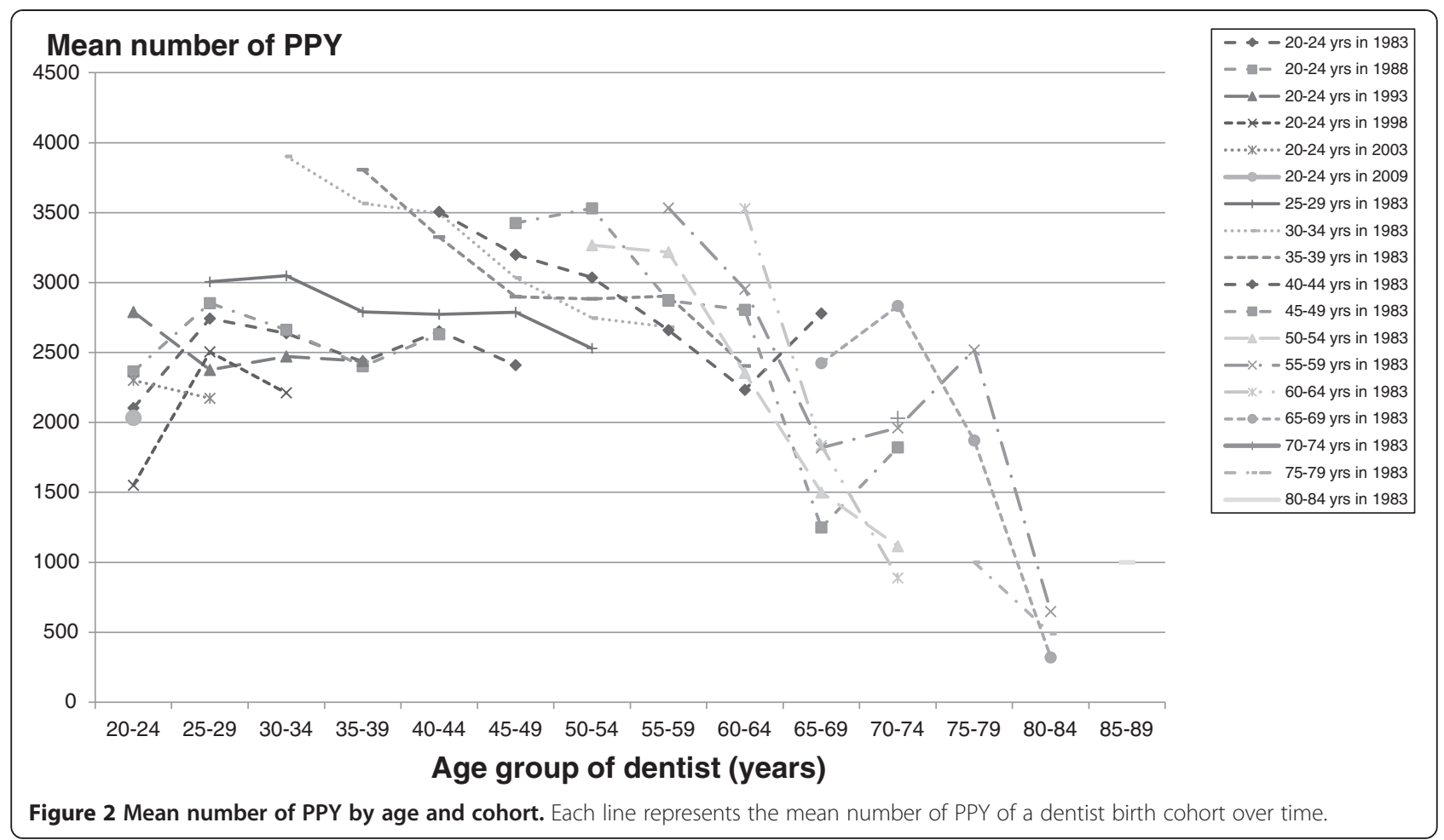

in 2003 had negative parameter estimates indicating lower PPY.

In summary, the cross-sectional age curve from the age-period model shows that the younger age dentists (20-29 years) and older dentists (65-74 and 80-84 years) have lower PPY than middle-aged dentists. The longitudinal age curve from this age-cohort model shows intracohort ageing effects of declining PPY over time within cohorts aged 45-84 years. Cohort parameters from the age-cohort model generally show higher PPY among earlier cohorts, and lower PPY among more recent cohorts.

\section{Discussion}

The present study investigated time trends and estimated age, period and cohort effects in patient visits supplied per dentist per year. The findings of the study have shown that the mean number of PPY decreased across most age groups of dentists over time, and the younger age dentists

Table 3 R-squared and P-values from goodness-of-fit tests from general linear regression analyses

\begin{tabular}{lccccc}
\hline Models & Number & $\begin{array}{c}\text { Degrees of } \\
\text { freedom }\end{array}$ & $\begin{array}{c}\mathbf{R} \\
\text { squared }\end{array}$ & $\begin{array}{c}\mathbf{F} \\
\text { value }\end{array}$ & $\mathbf{P}>\mathbf{F}$ \\
\hline Age & 2776 & 13 & 0.039 & 8.6 & $<0.0001$ \\
Age-drift & 2776 & 14 & 0.099 & 21.6 & $<0.0001$ \\
Age-period & 2776 & 18 & 0.102 & 17.4 & $<0.0001$ \\
Age-cohort & 2776 & 29 & 0.111 & 11.8 & $<0.0001$ \\
Age-period-cohort & 2776 & 33 & 0.115 & 10.8 & $<0.0001$ \\
\hline
\end{tabular}

(20-29 years) and older dentists (65-74 and $80-84$ years) have lower PPY than middle-aged dentists. There were intra-cohort ageing effects of declining PPY over time within cohorts aged 45-84 years, and higher PPY among earlier cohorts, and PPY was lower among more recent cohorts.

This longitudinal study was from a national survey and samples were selected randomly from a comprehensive sampling frame and achieved about 70 per cent response rates at each wave. Because the majority of dentists in Australia were from general practice and were from the private sector $[3,6,13]$, the data was weighted to reflect the age and sex distribution of private general practitioners in Australia. Therefore, the results can be generalized to

Table $4 \mathrm{~F}$ and $\mathrm{P}$ values from F-test and general linear regression analyses

\begin{tabular}{lcccccc}
\hline & \multicolumn{3}{c}{ Degrees of freedom } & F value & P-value \\
\cline { 2 - 5 } Models & $\mathbf{k}$ & $\mathbf{m}$ & $\mathbf{n}-\mathbf{k}-\mathbf{m}-\mathbf{1}$ & & \\
\hline Age \& age drift & 13 & 13 & 2761 & 9.66 & $<0.005$ \\
Age drift \& age-period & 14 & 14 & 2756 & 8.88 & $<0.005$ \\
Age drift \& age-cohort & 14 & 14 & 2744 & 13.96 & $<0.005$ \\
$\begin{array}{l}\text { Age-period-Cohort } \\
\text { \& age-Cohort }\end{array}$ & 29 & 29 & 2741 & 5.64 & $<0.005$ \\
$\begin{array}{l}\text { Age-period-Cohort } \\
\text { \& age-Period }\end{array}$ & 18 & 18 & 2740 & 11.52 & $<0.005$ \\
\hline
\end{tabular}

Notes: $\mathbf{n}$ was number of observations (see Table 3); $\mathbf{k}$ was degrees of freedom in the general linear regression model; $\mathbf{m}$ was additional variables, such as drift, age, period and cohort or their combination. 
Table 5 Age-period and age-cohort models of PPY

\begin{tabular}{|c|c|c|c|c|c|c|}
\hline & \multicolumn{3}{|c|}{ Age-period model } & \multicolumn{3}{|c|}{ Age-cohort model } \\
\hline & $\begin{array}{l}\text { Parameter } \\
\text { estimate }\end{array}$ & SE & $\mathbf{P}$ & $\begin{array}{l}\text { Parameter } \\
\text { estimate }\end{array}$ & SE & $P$ \\
\hline \multicolumn{7}{|l|}{$\begin{array}{l}\text { Age } \\
\text { group }\end{array}$} \\
\hline $20-24$ & -575.72 & 167.87 & 0.0006 & -227.75 & 183.01 & 0.21 \\
\hline $25-29$ & -215.46 & 91.67 & 0.0188 & 21.21 & 97.33 & 0.83 \\
\hline $30-34$ & 0.00 & & & 0.00 & & \\
\hline $35-39$ & 81.60 & 91.10 & 0.3705 & -148.17 & 94.88 & 0.12 \\
\hline $40-44$ & 259.74 & 92.16 & 0.0049 & -163.53 & 99.14 & 0.10 \\
\hline $45-49$ & 168.54 & 94.43 & 0.0744 & -447.04 & 105.24 & $<.0001$ \\
\hline $50-54$ & 200.62 & 96.90 & 0.0385 & -623.58 & 112.21 & $<.0001$ \\
\hline $55-59$ & 197.47 & 104.71 & 0.0594 & -811.74 & 131.32 & $<.0001$ \\
\hline $60-64$ & -85.57 & 114.86 & 0.4563 & -1244.09 & 144.70 & $<.0001$ \\
\hline $65-69$ & -685.31 & 152.71 & $<.0001$ & -2006.21 & 185.03 & $<.0001$ \\
\hline 70-74 & -805.75 & 199.77 & $<.0001$ & -2291.59 & 245.17 & $<.0001$ \\
\hline $75-79$ & -465.99 & 341.60 & 0.1726 & -2067.34 & 392.57 & $<.0001$ \\
\hline $80-84$ & -2153.59 & 546.17 & $<.0001$ & -3731.77 & 620.80 & $<.0001$ \\
\hline $85-89$ & -2104.31 & 1170.04 & 0.0722 & -2054.34 & 1167.05 & 0.08 \\
\hline \multicolumn{7}{|l|}{ Period } \\
\hline 1983 & 993.34 & 85.61 & $<.0001$ & & & \\
\hline 1988 & 706.21 & 79.16 & $<.0001$ & & & \\
\hline 1993 & 409.65 & 80.80 & $<.0001$ & & & \\
\hline 1998 & 188.70 & 79.83 & 0.0182 & & & \\
\hline 2003 & 107.99 & 76.62 & 0.1588 & & & \\
\hline 2009 & 0.00 & & & & & \\
\hline \multicolumn{7}{|l|}{ Cohort } \\
\hline $80-84$ (a) & - & & - & 648.55 & 958.64 & 0.50 \\
\hline 75-79 (a) & - & & - & 1267.25 & 686.92 & 0.07 \\
\hline $70-74$ (a) & - & & - & 1393.63 & 356.46 & $<.0001$ \\
\hline $65-69$ (a) & - & & - & 1223.43 & 274.77 & $<.0001$ \\
\hline $60-64(a)$ & - & & - & 1175.36 & 161.98 & $<.0001$ \\
\hline 50-59 (a) & - & & - & 772.51 & 153.49 & $<.0001$ \\
\hline 45-49 (a) & - & & - & 833.53 & 137.42 & $<.0001$ \\
\hline 40-44 (a) & - & & - & 690.22 & 117.07 & $<.0001$ \\
\hline $35-39$ (a) & - & & - & 551.33 & 102.35 & $<.0001$ \\
\hline $30-34$ (a) & - & & - & 545.69 & 92.84 & $<.0001$ \\
\hline $25-29$ (a) & - & & - & 0.00 & & \\
\hline $20-24$ (a) & - & & - & -330.88 & 89.43 & 0.00 \\
\hline $20-24$ (b) & - & & - & -352.63 & 102.26 & 0.00 \\
\hline $20-24$ (c) & - & & - & -539.13 & 114.43 & $<.0001$ \\
\hline $20-24$ (d) & - & & - & -778.79 & 131.20 & $<.0001$ \\
\hline $20-24$ (e) & - & & - & -847.71 & 162.35 & $<.0001$ \\
\hline $20-24$ (f) & & & & -793.63 & 455.75 & 0.08 \\
\hline
\end{tabular}

Note: $a=$ period 1 (1983), $b=$ period 2 (1988), $c=$ period 3 (1993), $d=$ period 4 (1998), e = period 5 (2003), $f=$ period 6 (2009). represent the main Australian dentist context of private general practice.

These findings point to a fundamentally different pattern of work for younger cohorts of dentists than older dentist cohorts. The findings show that younger cohorts are providing fewer patients visits each working year, and this work pattern appears to be relatively stable over time as they move into middle age. Previous reports have shown that the trend towards fewer patient visits was related to increased provision of services per visit and a shift in the types of services provided [1].

There are many factors that may influence and impact on PPY. The increasing proportion of female dentists (from 10\% in 1980 to $33 \%$ in 2009) can have a substantial influence on total aggregate capacity to provide dental services. Similarly, the proportion of female dentists increased in some other western developed countries, such as in Canada (from 17\% in 1991 to $37 \%$ in 2008) [14] and America (from 3\% in 1980 to $19 \%$ in 2000) [15]. This is because female dentists were undertaking more part time work [3], and taking more career breaks than male dentists [16-18]. The average worked hours per week decreased (from 39 hours in 2000 to 37 hours in 2009). The percentage working in solo private practice decreased (from 44\% in 2000 to $29 \%$ in 2009) to reflect a more flexible working pattern, such as solo with assistant, partnership and associateship arrangements [3].

Retaining more natural teeth in middle and older aged adults may consequently result in an increased burden of dental disease in older mouths [19] that may lead to demand for dental services. More complex dental treatment needs may lead to increased length of dental appointments, resulting in lower PPY. For instance, more endodontic and crown and bridge services have been associated with trends towards greater retention of teeth among adults $[1,19]$, as well as age-related oral diagnoses and insurance status [20,21].

Age-period-cohort models provide a formal framework to guide the analysis through an explicit consideration of all effects with assessing goodness-of-fit of models. Using the modelling approach to analyse age, period and cohort effects provides information to understand the time trends and inter-related time-dependent variables of age, period and cohort effects.

These findings are important to labour force planning in relation to the capacity to supply dental services. Australia's National Oral Health Plan included consideration of a sufficient, sustainable and appropriately skilled labour force to meet identified oral health needs across the Australian population [22] while the National Advisory Council on Dental Health conclude that advancement of foundational activities (such as those relating to the dental labour force) was integral to dental services delivery [23]. A review of Australian government health labour force programs noted 
the importance of data to inform dental policy debate [24]. Previous projections of the dental labour force in Australia have noted the importance of supply of dental visits to capacity to supply services [25]. Health Workforce Australia is investigating the number and mix in the oral health workforce to meet the changing demographics and policy requirements to 2025 [26].

The synthetic cohort approach used in this study was representative in terms of cross-sectional estimates, rather than being based on longitudinal changes. PPY was a key marker of practice activity. However, the component variables of HPY and PPH were not explicitly modelled. For instance, increased numbers of services per visit over time could decrease PPH [1], and an increase in the number of dentists per practice, dental assistants per practice, or the size of private practice (single handed or group) could reduce HPY. The age-period-cohort approach suffers from a confounding of age, period and cohort effects. This confounding makes the separation of age, period and cohort effects difficult unless all comparisons are pronounced and consistent [5]. Despite these limitations, this study of age, period and cohort effects in relation to patient dental visits in Australia is significant to future planning of the dental labour force in Australia.

\section{Conclusion}

The capacity of dentists to supply services might be influenced by age, period and cohort effects. Understanding dentists' capacity to supply dental services over time is a key element in the process of planning for the future. The study found a period effect of declining PPY over the observation period. More recent cohorts of dentists provided lower numbers of PPY than earlier cohorts at similar ages, but the provision of PPY among these younger cohorts appeared to be stable as they moved into middle age.

\section{Abbreviations}

HPY: Hours per dentist per year; PPH: Patient visits per dentist per hour; PPY: Patient visits per dentist per year.

\section{Competing interests}

The authors have no competing interests to declare.

\section{Authors' contributions}

XJ performed analyses and drafted the manuscript. DSB was involved in interpretation of data and contributing of drafting of the manuscript. AJS was involved in developing the project and revising the manuscript. The authors have read and approved the manuscript.

\section{Acknowledgments}

The Longitudinal Study of Dentists' Practice Activity has been supported by the Australian Government Department of Health and Ageing, the National Health and Medical Research Council (NHMRC), and the Australian Institute of Health and Welfare (AlHW). This paper was written with support from a Career Development Award (627037) and CRE (1031310) from the NHMRC. The contents are solely the responsibility of the administering institution and authors, and do not reflect the views of NHMRC.
Received: 4 July 2013 Accepted: 20 December 2013

Published: 10 January 2014

\section{References}

1. Brennan DS, Spencer AJ: Trends in service provision among Australian private general dental practitioners over a 20 -year period. Int Dent J 2006, 56:215-223.

2. Spencer AJ, Brennan DS, Szuster FSP: Trends in work effort among private general dental practitioners. Int Dent J 1994, 44:223-229.

3. Chrisopoulos S, Nguyen T: Trends in the Australian dental labour force, 2000 to 2009: dental labour force collection, 2009. Australian Institute of Health and Welfare: Canberra; 2012.

4. Brennan DS, Spencer AJ: Practice activity trends among Australian private general dental practitioners: 1983-84 to 1998-99. Int Dent J 2002, 52:61-66.

5. Bravo M: Age-period-cohort analysis of dentist use in Spain from 1987 to 1997: an analysis based on the Spanish National Health Interview Surveys. Eur J Oral Sci 2001, 109:149-154.

6. Brennan DS, Ryan P, Spencer AJ, et al: Dental service rates: age, period, and cohort effects. Community Dent Health 2000, 17:70-78.

7. Clayton D, Schifflers E: Models for temporal variation in cancer rates. II: age-period-cohort models. Stat Med 1987, 6:469-481.

8. Spencer AJ, Szuster FSP, Brennan DS: Service-mix provided to patients in Australian private practice. Aust Dent J 1994, 39:316-320.

9. Brennan DS, Spencer AJ, Szuster FSP: Service provision trends between 1983-84 and 1993-94 in Australian private general practice. Aust Dent J 1998, 43:331-336.

10. Brennan DS, Spencer AJ: Service provision trends among Australian private general dental practitioners: $1983 / 84$ to $1998 / 99$. Int Dent J 2003, 53:145-152

11. Polissar L, Diehr P: Regression analysis in health services research: the use of dummy variables. Med Care 1982, 20:959-966.

12. Clayton D, Schifflers E: Models for temporal variation in cancer rates. I: age-period-cohort models. Stat Med 1987, 6:469-481.

13. Teusner DN, Spencer AJ: Dental labour force, Australia 2000. Australian Institute of Health \& Welfare: Canberra; 2003.

14. McKay JC, Quinonez CR: The feminization of dentistry: implications for the profession. J Can Dent Assoc 2012, 78:1-7.

15. Solomon ES: The future of Dentistry. http://www.dentaleconomics.com/ articles/print/volume-95/issue-2/features/the-future-of-dentistry.html.

16. Brennan DS, Spencer AJ, Szuster FSP: Differences in time devoted to practice by male and female dentists. Br Dent J 1992, 172:348-349.

17. Brennan DS, Balasubramanian M, Spencer AJ: Practice profiles of male and female dentists in Australia. Aust Dent J 2011, 56:97-99.

18. Ayers KM, Thomson WM, Rich AM, et al: Gender differences in dentists' working practices and job satisfaction. J Dent 2008, 36:343-350.

19. Spencer AJ: Putting the population back into oral health; decoupling oral health improvement from clinical dental practice. Community Dent Oral Epidemiol 2012, 40(Suppl 2):5-11.

20. Brennan DS, Spencer AJ, Szuster FSP: Service provision patterns by main diagnoses and characteristics of patients. Community Dent Oral Epidemiol 2000, 28:225-233.

21. Brennan DS, Spencer AJ, Szuster FSP: Insurance status and provision of dental services in Australian private general practice. Community Dent Oral Epidemiol 1997, 25:423-428.

22. NACOH: Healthy mouths healthy lives: Australia's National Oral Health Plan 2004-2013. Adelaide: National Advisory Committee on Oral Health, Australian Health Ministers' Advisory Council; 2004.

23. National Advisory Council on Dental Health: Report of the National Advisory Council on Dental Health. Canberra: Department of Health and Ageing; 2012.

24. Mason J: Review of Australian Government Health Workforce Programs. Canberra: Department of Health and Ageing; 2013.

25. ARCPOH: Dental labour force projections 2005 to 2020: the impact of new regional dental schools. Aust Dent J 2008, 53:292-296.

26. Health Workforce Australia: Health Workforce 2025 - Oral Health, Background Paper. Adelaide: Health Workforce Australia; 2013.

doi:10.1186/1472-6963-14-13

Cite this article as: Ju et al:: Age, period and cohort analysis of patient dental visits in Australia. BMC Health Services Research 2014 14:13. 